\title{
The Allele Distribution for the rs7250346 SNP in SLC5A5 among Saudis
}

Abdullah T. Eissa.'

\begin{abstract}
Background: The sodium/iodide cotransporter solute carrier family 5 member 5 ( $\left.\mathrm{SLC}_{5} \mathrm{~A}_{5}\right)$ is found in the basolateral cell membrane of thyroid follicular epithelial cells as a sodium iodide symporter. It helps in the secretion of triiodothyronine (T3) and tetraiodothyronine (T4). Polymorphisms in SLC5A5 result in hypothyroidism. The aim of the study is to estimate the frequency of rs 7250346 , a single nucleotide polymorphism (SNP) associated with thyroid disease, in a Saudi population and to compare it to other populations. Methods: Two hundred and forty Saudi patients from King Faisal Specialist Hospital and Research Centre provided samples for the genetic analysis. Samples were genotyped for target SNPs by real time polymerase chain reaction (PCR), and the resultant rs 7250346 frequency in the Saudi population was compared to other populations using HapMap. Results: Out of the 240 Saudi samples, $64 \%$ had C genotype and $35 \%$ had G genotype in the rs7250346. Conclusion: The Saudi frequency of the rs7250346 SNP of SLC5A5 differs from that of European, Chinese, Japanese, or Sub-Saharan populations in HapMap (http://hapmap.org).
\end{abstract}

Keywords: SLC5A5 protein, human; Hypothyroidism; Polymorphism, Genetic (Source: MeSH-NLM).

About the Author: Abdullah Talat Eissa is a final-year medical student at king Saud bin Abdulaziz University for Health Sciences, Riyadh, Saudi Arabia. He is also a pharmacist who graduated from king Saud University in 2010
Submission: Apr 5, 2015

Acceptance: Aug 25, 201

Publication: Dec 31, 2015

Process: Peer-reviewed

\section{Introduction}

The nature of many diseases and their occurrence vary from one population to another according to their genetic backgrounds. These genetic variations are influenced by mutations, natural selections, migrations and genetic drifts.' When the genomic sequence is altered in less than $1 \%$ of population, compared to the referenced sequence, the mutation will occur. However, if the alteration on a specific genome is present in $\geq 1 \%$ of population, the polymorphism becomes the source of this variation. Moreover, the most common genetic variation in humans is caused by single nucleotide modification. The Single Nucleotide Polymorphism (SNP) presents if $\geq 1 \%$ of population possess a change in a nucleotide. ${ }^{2}$ Determining the SNP is useful to explain the susceptibility of an individual to disorders, vaccines and medications. Some genetic diseases follow simple patterns of transmissions (simple Mendelian diseases), which are caused by a single SNP. Nonetheless, for complex genetic diseases, other factors in addition to SNPS play roles to manifest diseases such as the environmental factor. ${ }^{3}$

The SLC5A5 gene encodes the sodium iodide symporter. It is located at 19p13.11. The sodium-iodide symporter (NIS) is an integral membrane glycoprotein with 634 amino acids and molecular weight of $69 \mathrm{~K} \mathrm{Da}$, it consists of 15 exons spanning $23202 \mathrm{bp}$ and 13 transmembrane domains and an extracellular $\mathrm{N}$-terminal. $\mathrm{SLC}_{5} \mathrm{~A}_{5}$ is found in the basolateral cell membrane of thyroid follicular epithelial cells. ${ }^{4}$ In 1996, it was sequenced from rats followed by human thyroid, ${ }^{5}$ while the description of exon-intron organization occurred in 1997.6

Triiodothyronine $\left(\mathrm{T}_{3}\right)$ and tetraiodothyronine $\left(\mathrm{T}_{4}\right)$ are thyroid hormones which are synthesized when $\mathrm{SLC}_{5} \mathrm{~A}_{5}$ concentrates
I in the thyroid.? The iodine can be taken with a trace amount from food supplement. It can be used for the diagnostic scintigraphic imaging or as radio iodide therapy when $\mathrm{SLC}_{5} \mathrm{~A} 5$ accumulate iodide in the thyroid gland. ${ }^{8}$ NIS is organified into the thyroid hormone precursor thyroglobulin by thyroid peroxidase in the presence of hydrogen peroxide $\left(\mathrm{H}_{2} \mathrm{O}\right)$. It's regulated by thyroid stimulating hormone (TSH) under control of the hypothalamic-pituitary axis. ${ }^{9}$

Polymorphisms in SLC5A5 have also been related to a congenital iodide transport defect (ITD), which can cause hypothyroidism. ${ }^{10}$ The $\mathrm{C} .1060 \mathrm{~A}>\mathrm{C}$ variant associated with congenital hypothyroid goiter leads to failure of iodide transport. ${ }^{11,12}$ Homozygosity for the same variant in nucleotide 1060 (euthyroid goiter) will lead to a defect in iodide transport. ${ }^{13,14}$ The C. $1146 C>C$ variant (hypothyroid goiter) will lead to NIS without detectable bioactivity and low expression, ${ }^{15}$ and the $c .1629 \mathrm{C}>\mathrm{A}$ variant (congenital hypothyroid goiter) will give minimal iodide uptake. ${ }^{16} \mathrm{~A} 6192 \mathrm{bp}$ deletion in exon 3-7 (congenital hypothyroid goiter) will result in total failure of iodide transport. ${ }^{17}$ This SNP of SLC5A5 was studied because it has previously been shown to have a significant association with thyroid diseases. ${ }^{18}$ In addition, it is an intron that is identified as rs7250346, and located on chromosome 19:17893553, the polymorphism is a C/C transversion substitution. Given that there are higher incidences of thyroid diseases in the Saudi population, ${ }^{19,20}$ it is plausible that some of this difference may stem from genetic polymorphisms. The different distribution of the genotypes may give some explanations of the incidence of thyroid related diseases in this population. The aim of this study is to measure the frequency

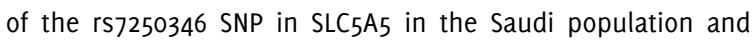
compare it with other populations. 


\section{Methods \\ Study Population}

A cohort of 240 healthy Saudi individuals from the outpatient clinics, who came for routine check-ups or following mild conditions that are not interfere with the purpose of the study such as controlled diabetes mellitus, at King Faisal specialist Hospital and Research Centre was admitted to the study. Individuals who had normal blood, biochemistry, hormones (including TSH) and coagulation laboratory results were recruited. Patients who had liver impairment, renal insufficiency, mental instability or who were on multiple medications were excluded.

\section{DNA Isolation}

Five milliliters of blood was sampled from each participant after obtaining their written consent. DNA was isolated using the Puregene DNA isolation kit according to manufacturer's protocol (Qiagen Sciences, Maryland, USA). Briefly, erythrocytes were lysed in RBC lysis buffer, and the proteins were removed by precipitation in TCA buffer. The DNA was subsequently isolated by precipitation in alcohol, quantified and stored at $-80^{\circ} \mathrm{C}$ if not required immediately.

\section{Real-Time PCR Genotyping}

Samples were genotyped for target SNPs by real time PCR using the $7900 \mathrm{HT}$ Sequence Detection System (ABI, Foster City, CA, USA). Primers and TaqMan probes were designed using the Primer Express software $\mathrm{V} 2.0$ ( $\mathrm{ABI}$, Foster City, CA, USA) and obtained from Applied Biosystems (Warrington, UK). The fluorogenic probes, bearing a suitable reporter dye on the $5^{\prime}$ - end and a quencher dye on the 3 '-end, hybridize to the specific sequence to be amplified by the PCR primers. The quencher prevents fluorescent emission from the reporter dye when the probe is intact. When the probe is cleaved by the polymerase exonuclease activity in the extension phase of $P C R$, there is increased fluorescent emission from the reporter which is no longer in close proximity to the quencher.

One probe, for allele 1 , is labeled with VIC dye and the other, for allele 2, with FAM dye at the $5^{\prime}$-end. Serial dilutions of the probes were run to determine the optimal working concentration. For each well of a 96-well reaction plate a $25 \mu \mathrm{l}$ reaction was prepared by mixing $5 \mu$ l containing 5ong DNA, $12.5 \mu \mathrm{l}$ of 2x universal mix (Eurogentec, liege Science Park, 4102 Seraing, Belgium), $1.25 \mu \mathrm{l}$ of $20 \mathrm{x}$ probe Assay mix and $6.25 \mu \mathrm{l}$ of $1 \mathrm{x}$ Tris EDTA buffer. Three no-template controls were included for each plate. The thermal profile for amplification was a 1st cycle at $50^{\circ} \mathrm{C}$ for 2 minutes, and $95^{\circ} \mathrm{C}$ for $10 \mathrm{~min}$, followed by 40 cycles of $94^{\circ} \mathrm{C}$ for $15 \mathrm{sec}$, and $60^{\circ} \mathrm{C}$ for $30 \mathrm{sec}$. The plate wells were scanned for fluorescence resonance energy transfer (FRET) signal in the $7900 \mathrm{HT}$ sequence detection system, and the data analyzed using SDS 2.0 software (ABI, Foster City, CA, USA). Excel program was used to analyze the data. The Saudi genotypes of rS7250346 SNP in SLC5A5 were compared with other populations, particularly, Sub-Saharan Africans, Chinese, Japanese and Europeans, which are included in the HapMap project. This project studied 11 populations in three phases. The three collections consist of 1,301 samples in addition to the original 270 samples used in the first two phases. Furthermore, this resource was aimed to identify the common patterns of human DNA sequence variation. According to the HapMap samples, all participants provided informed consents.

\section{Results}

In this study, the total population included 240 patients from the outpatient clinics at King Faisal Specialist Hospital and Research Centre, comprised of $146(61 \%)$ females and $94(39 \%)$ males. The mean age of the subjects was $44+/-15$ years. Two alleles were examined for each subject to create a total of 480 alleles samples. Out of 480 Saudi alleles, 308 (64\%) had a C genotype for the rS7250346 SNP in SLC5A5 and 164 (35.4\%) had a $G$ genotype (Figure 1).

Figure 1. Prevalence of $\mathrm{rS} 7250346$ of SLC5A5 Cene by Ethnicity.

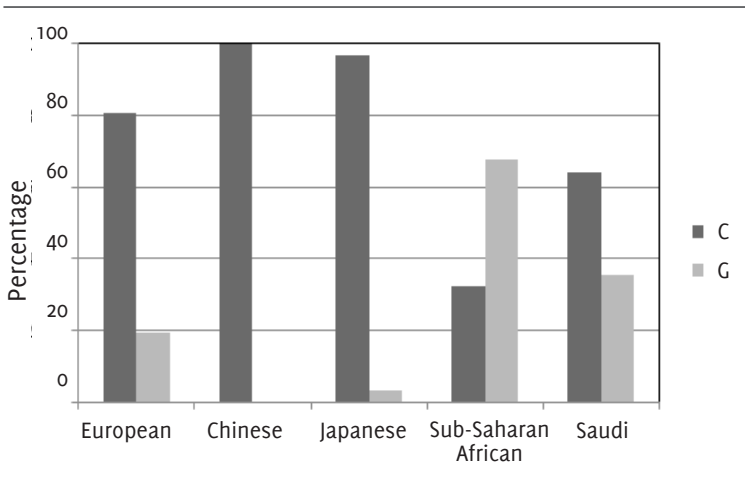

\section{Discussion}

The goal of the study was to determine the differences between Saudi and other populations for the rs7250346 SNP in SLC5A5. However, a possible limitation of this study is that NIS was the only SNP analyzed in this population. It is an intron that can lead to interesting molecular biology discussion because it varies between different geographical populations and it is associated with different conditions. This SNP is associated with the thyroid gland, and some variants are linked to functional abnormalities. Some studies suggested that certain polymorphisms might lead to hypothyroidism, ${ }^{10}$ congenital hypothyroid goiter, ${ }^{11}$ and hypothyroidism. ${ }^{15}$ Nevertheless, studies on NIS are scarce and there is insufficient data to link a variant of this gene to diseases in certain populations.

In the Saudi population, the incidence of thyroid cancer increased dramatically between 2000 and 2010 where it was responsible for $9 \%$ of all malignancies in one Saudi hospital, compared to $2.9 \%$ in U.S. In addition, in King Faisal Specialist Hospital at Research Centre, it became the second most common cancer among females. ${ }^{19,20}$ For that reason, this study sought to investigate how the Saudi $\mathrm{SLC}_{5} \mathrm{~A}_{5}$ differs from other populations, which may help researchers understand if this SNP has a role in the high incidence of other thyroid disorders, including thyroid cancer, in this population.

This study demonstrated that there is a marked difference between the Saudi allele frequency and other populations for SNP rS7250346. Figure 1 shows the genotype in Saudi population ( $C=64 \%$ and $C=35.4 \%$ ), Also it represents the same gene in HapMap (http://hapmap.org) with other different populations, namely, European, Japanese, Chinese and Sub-Saharan African, which are not similar to the Saudi population gene. In the Chinese population, $100 \%$ possessed the $C$ genotype while it occurred in $97 \%$ of the Japanese population. The $\mathrm{G}$ genotype occurred in $20 \%$ Europeans and C genotype in $80 \%$. The distribution of the genotypes in Sub-Saharan African is $\mathrm{C}=32.5 \%$ and 
$\mathrm{G}=67.5 \%$. The variations in the prevalence of thyroid abnormalities and how they are associated with genetic polymorphisms can be observed if congenital hypothyroidism is taken as an example, where it is correlated with the hereditary in $15 \%$ of the cases. Importantly, it was found that defects in NIS, which includes thyroid hormone transport abnormalities, was considered as one of the causes of this illness. ${ }^{21}$

According to a Chinese screening study, the prevalence of congenital hypothyroidism is increasing, where 6,505 of neonates were diagnosed as congenital hypothyroidism out of 13,229,242 Chinese newborns from 1985 to $2006 .{ }^{22}$ Also, a New York screening program from 2000 to 2003 published that congenital hypothyroidism differed among four ethnicities, and it showed that Caucasian and Black infants had lower incidence of congenital hypothyroidism when compared to Hispanic and Asian populations. Congenital hypothyroidism was diagnosed in 1/1815 births among Caucasian infants, 1/1902 of Black infants, 1/1559 of Hispanic infants and 1/1016 of Asians infants. ${ }^{23}$

Unfortunately, there are not enough studies to determine an ethnic pattern for SLC5A5 in the Saudi population. Further studies are warranted to discover the gene differences between populations. Furthermore, research into the genetic composition of the Saudi population is needed to provide an appropriate estimation of gene patterns. Studying this SNP may have some implications for future research, clinical practice and public health policy. Saudi SLC5 55 may allow researchers to investigate its association with hypothyroidism, which may prevent unnecessary prescription of Thyroxin to treat an idiopathic condition, or even prevent it in the first place. Moreover, finding how Saudi genotypes differ from other ethnic groups may provide some clues about why thyroid abnormalities are widely diagnosed in the Arabia Peninsula. 


\section{References}

1. Torkamani A, Pham P, Libiger O, Bansal V, Zhang G, Scott-Van Zeeland AA, et al. Clinical implications of human population differences in genome-wide rates of functional genotypes. Front Genet. 2012 Nov 1;3:211.

2. Carlson CS, Eberle MA, Rieder MJ, Yi Q, Kruglyak L, Nickerson DA. Selecting a maximally informative set of single-nucleotide polymorphisms for association analyses using linkage disequilibrium. Am J Hum Genet. 2004 Jan;74(1):106-20.

3. Carlson CS, Eberle MA, Kruglyak L, Nickerson DA. Mapping complex disease loci in whole-genome association studies. Nature. 2004 May 27;429(6990):446-52. 4. Di Bernardo J, Rhoden KJ. SLC5A5 (solute carrier family 5 (sodium iodide symporter), member 5) (19p13.11). Atlas Cenet Cytogenet Oncol Haematol. 2010 June; 14(6):581-7.

5. Smanik PA, Liu Q, Furminger TL, Ryu K, Xing S, Mazzaferri EL, et al. Cloning of the human sodium iodide symporter. Biochem Biophys Res Commun. 1996 Sep13;226(2):339-45.

6. Smanik PA, Ryu KY, Theil KS, Mazzaferri EL, Jhiang SM. Expression, exon-intron organization, and chromosome mapping of the human sodium iodide symporter. Endocrinology. 1997 Aug;138(8):3555-8.

7. Dohán 0 , De la Vieja A, Paroder V, Riedel C, Artani M, Reed M, et al. The sodium/iodide symporter (NIS): characterization, regulation, and medical significance. Endocr Rev. 2003 Feb;24(1):48-77.

8. Dohan 0, Gavrielides MV, Ginter C, Amzel LM, Carrasco N. Na(+)/I(-) symporter activity requires a small and uncharged amino acid residue at position 395. Mol Endocrinol. 2002 Aug;16(8):1893-902.

9. Matsuda A, Kosugi S. A homozygous missense mutation of the sodium/ iodide symporter gene causing iodide transport defect. J Clin Endocrinol Metab. 1997 Dec;82(12):3966-71.

10. Fujiwara H, Tatsumi K, Miki K, Harada T, Okada S, Nose O, et al. Recurrent T354P mutation of the $\mathrm{Na}+/ \mathrm{I}$ - symporter in patients with iodide transport defect. J Clin Endocrinol Metab. 1998 Aug;83(8):2940-3.

11. Fujiwara H, Tatsumi K, Miki K, Harada T, Miyai K, Takai S, et al. Congenital hypothyroidism caused by a mutation in the $\mathrm{Na}+/ \mathrm{l}$ - symporter. Nat Genet. 1997 Jun;16(2):124-5.

12. Levy 0 , Ginter CS, De la Vieja A, Levy D, Carrasco N. Identification of a structural requirement for thyroid $\mathrm{Na}+/ \mathrm{l}$ - symporter (NIS) function from analysis of a mutation that causes human congenital hypothyroidism. FEBS Lett. 1998 Jun 5;429(1):36-40.

13. Böttcher $Y$, Eszlinger M, Tönjes A, Paschke R. The genetics of euthyroid familial goiter. Trends Endocrinol Metab. 2005 Sep;16(7):314-9.

14. Konbel M, Medeiros-Neto $G$. An outline of inherited disorder of the thyroid hormone generating system. Thyroid. 2003 Aug;13(8):771-801.

15. Pohlenz J, Rosenthal IM, Weiss RE, Jhiang SM, Burant C, Refetoff S. Congenital hypothyroidism due to mutations in the sodium/iodide symporter. Identification of a nonsense mutation producing a downstream cryptic 3' splice site. J Clin Invest. 1998 Mar1;101(5):1028-35.

16. Kosugi S, Sato $Y$, Matsuda A, Ohyama Y, Fujieda $K$, Inomata $H$, et al. High prevalence of $\mathrm{T} 354 \mathrm{P}$ sodium/iodide symporter gene mutation in Japanese patients with iodide transport defect who have heterogeneous clinical pictures. J Clin Endocrinol Metab. 1998 Nov;83(11):4123-9.

17. Kosugi S, Okamoto H, Tamada A, Sanchez-Franco F. A novel peculiar mutation in the sodium/iodide symporter gene in Spanish siblings with iodide transport defect. J Clin Endocrinol Metab. 2002 Aug;87(8):3830-6.

18. Al-Rasheed MM, Alzahrani AS, Macadam A, Overall A, Gard P, Dzimiri N. The potential role of the sodium symporter gene polymorphism in the development of differentiated thyroid cancer. Gene. 2015 Nov 10;572(2):163-8. 19. Hussain F, Iqbal S, Mehmood A, Bazarbashi S, ElHassan T, Chaudhri N. Incidence of thyroid cancer in the Kingdom of Saudi Arabia, 2000-2010. Hematol Oncol Stem Cell Ther. 2013 Jun;6(2):58-64.

20. Malabu UH, Alfadda A, Sulimani RA, Al-Rubeaan KA, Al-Ruhaily AD, Fouda MA, et al. Graves' disease in Saudi Arabia: a ten-year hospital study. J Pak Med Assoc. 2008 Jun; 58(6):302-4.

21. Grasberger H, Refetoff $S$. Genetic causes of congenital hypothyroidism due to dyshormonogenesis. Curr 0pin Pediatr. 2011 Aug;23(4):421-8.

22. Xu YH, Qin YF, Zhao ZY. [Retrospective study on neonatal screening for congenital hypothyroidism and phenylketonuria in China in the past 22 years]. Zhonghua Er Ke Za Zhi. 2009 Jan;47(1):18-22.

23. Harris KB, Pass KA. Increase in congenital hypothyroidism in New York State and in the United States. Mol Genet Metab. 2007 Jul;91(3):268-77.

\footnotetext{
Acknowledgments

Special thanks to the Department of Medical Genetics at King Faisal Specialist Hospital and Research Centre for providing the laboratory and the samples for the research.

Conflict of Interest Statement a Funding

The Authors have no funding, financial relationships or conflicts of interest to disclose.

Author Contributions

Conception and design the work/idea, Collect data/obtaining results, Analysis and interpretation of data, Write the manuscript, Critical revision of the manuscript, Approval of the final version: ATE.

Cite as:

Eissa AT. The Allele Distribution for the rs7250346 SNP in SLC5A5 among Saudis. Int J Med Students. 2015 Sep-Dec;3(3):127-30.
} 